\title{
IMPROVING THE QUALITY OF HIV DATA
}

Sydney $M$ Bell $^{1,2}$, Peter $W$ Robertson ${ }^{\prime}$, L Ross Whybin ${ }^{7}$ and Mark J Ferson ${ }^{2}$

${ }^{t}$ Microbiology Department, The Prince of Wales Hospital

${ }^{2}$ Eastern Sydney Public Health Unit

$t$ is universally agreed that provision of timely and accurate epidemiological data on newly diagnosed cases of human immunodeficiency virus (HIV) infection is essential for the control of this disease. It would be reasonable to expect that information obtained from HIV testing laboratories, especially that concerning patients in the early stages of the disease, would be valuable in tracking and influencing the course of the epidemic.

In NSW, where the vast majority of this country's cases occur, confirmatory testing of positive HIV screening tests is performed in only four laboratories. Since the advent of HIV testing these laboratories have been the major source of data on all confirmed "new diagnoses" of HIV infection, and there has been an expectation that this would allow ready access to all laboratory data pertaining to confirmed "new diagnoses". However, despite this apparent advantage and the generous allocation of resources for HIV/AIDS surveillance to the NSW Health Department, the quality of data collected has been poor. A previous NSW Health Minister described it as "woeful".

In early 1992, when we reviewed the situation, we found an intolerable lack of basic and important information had been collected on the more than 15,000 "new diagnoses" of HIV contained in the National HIV Database. For example, in more than 18 per cent of the NSW cases the sex of the patient was unknown; nationally, age was not recorded in 24 per cent of cases, and in more than 40 per cent exposure category was not stated. At that time the information supplied to the NSW Health Department and in turn to the National HIV Database by laboratories was derived solely from information given by requesting practitioners on the HIV request form.

An examination of 400 request forms received by the Prince of Wales HIV Reference Laboratory in 1991 revealed a lack of information similar to that contained in the National HIV Database. Moreover, only one third of the request forms contained unique $2+2$ patient identifiers (i.e. at least the first two letters of each of the given name and surname), a further 20 per cent were identified by a clinic code number and the remainder were given simply a private code by the doctor or allocated a laboratory accession number. The paucity of identifying information made disaggregation of data virtually impossible by either the Reference Laboratory or NSW Health Department and cast doubt on the validity of the number of "new diagnoses" stated to be in the National HIV Database.

\section{IMPLEMENTATION OF THE CALL-BACK SYSTEM}

To improve the quality of data, a system of "call-back" to referring practitioners by mail/telephone was introduced from April 22, 1992, for patients whose specimens tested positive for the first time in the Prince of Wales Hospital HIV Reference Laboratory and who were not known to have been tested as HIV antibody positive elsewhere. Where the specimen had been sent by another laboratory, it was necessary to obtain the name and address of the original requesting medical officer to whom the questionnaire was to be sent and the patient identifier used by that doctor. In such cases, this initial task could be carried out only by telephoning the referring laboratory and was the most timeconsuming part of the call-back system.
Once the medical officer's details were obtained, a letter over the signature of the Medical Officer of Health for the Eastern Sydney Area Health Service was sent by certified mail to the referring doctor. The letter outlined the laboratory's obligation to supply information under the Public Health Act 1991 and sought the doctor's cooperation in obtaining this information. Enclosed with the letter was a notification form containing the 11 fields to be completed, with a reply-paid envelope addressed to the Medical Officer of Health. If no reply was received within one month a gentle reminder with another notification form and replypaid envelope was sent to the referring practitioner. All correspondence was contained in envelopes marked "Confidential" and return envelopes were delivered unopened to one of the senior officers in the HIV Reference Laboratory who entered the data and checked for possible duplication and inconsistencies. If no reply was received to the second letter after a further month had elapsed, the Director of the Eastern Sydney Public Health Unit telephoned and reminded the doctor to complete the form.

The data obtained were entered onto a database program designed for the purpose. This program is part of a PC network with strictly limited access.

\section{RESULTS}

In the 18 months from April 22, 1992 to October 31, 1993, 286 new diagnoses of HIV infection were confirmed in the HIV Reference Laboratory, Prince of Wales Hospital. In 13 cases all the fields were completed on the request form by the referring doctor and no further action was needed. In the remaining 273 cases it was necessary to initiate the callback procedure. The overall response to the call-back by referring practitioners was excellent, with a 100 per cent compliance with the scheme. Two hundred and forty-five (89.7 per cent) of the referring doctors returned the completed forms in response to the first letter, 19 ( 7 per cent) responded to the reminder letter while only nine needed to be prompted with a telephone call.

There were 11 fields requiring completion by the referring doctor and despite this expanded questionnaire the response was highly satisfactory. The fields least likely to be completed were the unique patient identifiers and the patient's home postcode (each 96.9 per cent complete) while the percentage completion of all other fields was 98 per cent or better. Table 9 compares the completeness of data supplied on the request forms sampled from 1991 and those obtained by call-back in 1992-93.

\section{DISCUSSION}

The successful call-back system was implemented without the allocation of additional resources to the Reference Laboratory or the PHU.

The advantage of having initially involved the senior staff was seen as the scheme progressed. Referring doctors recognised the involvement of the senior staff and responded accordingly. For example, in the few instances where they were unable to complete a field they generally included an unsolicited explanation of the cause of the omission. There has been a considerable reduction in the number of reminder letters required and in the past 12 months a telephone reminder has been needed only once. 


\begin{tabular}{|c|c|c|}
\hline \multicolumn{3}{|l|}{ TABLE 5} \\
\hline \multicolumn{3}{|c|}{$\begin{array}{l}\text { COMPLETENESS OF HIV DATA BEFORE AND } \\
\text { AFTER INSTITUTION OF 'CALL BACK' SYSTEM }\end{array}$} \\
\hline \multirow[t]{2}{*}{ Information } & ntage of each & eld completed \\
\hline & $\begin{array}{r}1991 \text { Request } \\
\text { forms }\end{array}$ & $\begin{array}{r}1992-93 \text { after } \\
\text { call back }\end{array}$ \\
\hline Unique patient identifier & 33 & 96.9 \\
\hline Patient's home postcode & 44 & 96.9 \\
\hline Sex & 84 & 100 \\
\hline Date of birth & 93 & 100 \\
\hline Exposure category & 54 & 99 \\
\hline Clinical status & 59 & 98.6 \\
\hline History of previous HIV test & 64 & 100 \\
\hline
\end{tabular}

It is still unclear why so many years elapsed before any real attempt was made to improve the quality of data collected on HIV infection. Perhaps it needed the force of the Public Health Act 1991 which made HIV infection a notifiable disease, although legislation has not been found necessary to achieve high quality data for other disease registries. However, two points became obvious to us soon after initiating the call-back system. The first was that there was no reluctance on the part of referring doctors to supply adequate information provided they were asked specifically for it and given a reasonable explanation for the request. Second, to be successful the call-back process needs to be a collaborative effort involving the testing laboratory and the PHU. This second observation is not surprising in view of the expertise and resources available in PHUs for collecting data on infectious diseases.

\section{EDITORIAL COMMENT}

The recently publicised case of patient-to-patient HIV transmission $^{1}$ has raised the question of whether this situation may have occurred again, before or since, and the ability of HIV/AIDS surveillance in NSW to detect such an event.

HIV testing that is readily available and free provides the potential for comprehensive surveillance. While there is an overriding need to ensure patient confidentiality, good quality data should include enough personal information to exclude the notification of the same individual for more than one positive test. This has been an insurmountable obstacle for most countries - Australia is one of only four known countries that routinely monitor and publish data on all HIV diagnoses. The others are New Zealand, the United Kingdom and Papua New Guinea. Considerable improvements have been made in NSW, although other laboratories have not been able to provide data as complete as that from Prince of Wales Hospital (POW). Figure 5 shows the impact of the introduction of the callback system by all laboratories in early 1992 . The proportion of cases where information on risk exposures is missing has been reduced from 52 per cent overall to 22 per cent in the year 1992-93.

Apart from missing data, two categories of exposure are of concern in monitoring unusual means of transmission unspecified heterosexual contact and the "unknown" category (figure 5). Reported unspecified heterosexual contact (where no more information on the source of infection is available or provided), may be a default category in some cases where another source of infection is not known or not revealed by the patient. The "unknown" category covers only a very small number of notifications which do not fall into existing categories, the majority being where common exposures are denied by the patient.

A national study of HIV surveillance data from January to October 1991 (before the call-back system began) collected more detail via a questionnaire on all notifications where

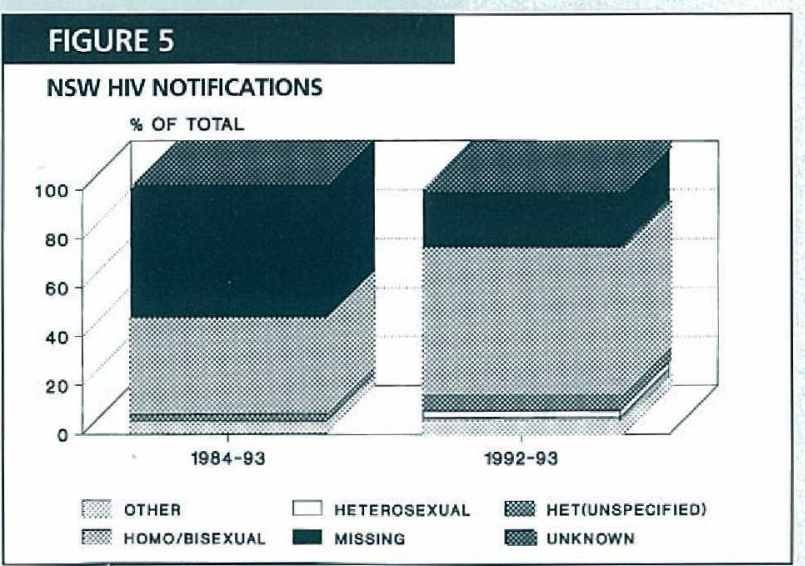

exposure was not reported as either male homosexual contact or blood transfusion ${ }^{2}$. Of questionnaires returned, the two categories of main interest were:

- those where exposure was originally missing 40 per cent were actually not new HIV diagnoses, 33 per cent were found to be new diagnoses actually due to male homosexual contact, and most of the remainder were found to be due to heterosexual contact; and

- those where exposure was originally reported as unspecified heterosexual contact - 26 per cent were old diagnoses and 16 per cent were new diagnoses due to male homosexual contact. Of those reported on the questionnaire as unspecified heterosexual contact, the doctor was satisfied with the patient's report in 64 per cent of cases.

Therefore, on further investigation, of those with either missing data on exposure or reported unspecified heterosexual contact, only 22 ( 25 per cent) remained in these categories after the questionnaire had been returned.

The HIV surveillance data from Prince of Wales Hospital for the period July 1, 1992 to September 30, 1993 are among the most complete available in NSW as laboratory callback was operating throughout this period. Of 188 notifications of new diagnoses, in 18 cases ( 9.6 per cent) the doctor was unable to ascertain the exposure category and 16 ( 8.5 per cent) reported unspecified heterosexual contact. Further, of the 2,673 NSW cases of AIDS notified to November 30 , 1993 , in $34(1.3 \%)$ the exposure category was not established and 29 were not interviewed.

There is considerable room for improvement. The Chief Health Officer has written to Area Chief Executive Officers and District General Managers about this issue. In future rates of missing information for all notifications will be expected to be of the same standard as that from POW. Relevant PHUs will be encouraged to assist as Eastern Sydney Area PHU does for POW. The study mentioned above was intended as a pilot of an ongoing method of improved data collection. From January 1994 all notifications, except those reporting male homosexual contact, will be followed up by questionnaire, to verify the report, collect more information and detect cases requiring further investigation. In cases where the mode of transmission has not been established, a formal investigation will begin, subject to patient consent. The first stage of the investigation will be a standard patient interview similar to that developed by the US Centers for Disease Control for investigating such cases.

1. Chant $\mathrm{K}$, Lowe $\mathrm{D}$, Rubin G et al. Patient-to-patient transmission of HIV in private surgical consulting rooms. Lancet 1993; 342:1548-9. 2. McDonald A. Personal communication, 1994. 